\title{
« Mi tótem tiene que ver con ese animal duro de cazar que corre con su propia gracia » Simanca Pushaina ou la poétique de la négociation
}

"Mi tótem tiene que ver con ese animal duro de cazar que corre con su propia gracia ». Simanca Pushaina or the poetics of negotiation. "Mi tótem tiene que ver con ese animal duro de cazar que corre con su propia gracia ». Simanca Pushaina o la poética de la negociación.

\section{Laura Lema Silva}

\section{CpenEdition}

1 Journals

Édition électronique

URL : http://journals.openedition.org/ideas/8527

DOI : $10.4000 /$ ideas.8527

ISSN : 1950-5701

Éditeur

Institut des Amériques

Référence électronique

Laura Lema Silva, « « Mi tótem tiene que ver con ese animal duro de cazar que corre con su propia gracia » Simanca Pushaina ou la poétique de la négociation », IdeAs [En ligne], 16 | 2020, mis en ligne le 01 octobre 2020, consulté le 18 octobre 2020. URL : http://journals.openedition.org/ideas/8527 ; DOI : https://doi.org/10.4000/ideas.8527

Ce document a été généré automatiquement le 18 octobre 2020.

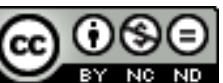

IdeAs - Idées d'Amériques est mis à disposition selon les termes de la licence Creative Commons Attribution - Pas d'Utilisation Commerciale - Pas de Modification 4.0 International. 


\title{
«Mi tótem tiene que ver con ese animal duro de cazar que corre con su propia gracia » Simanca Pushaina ou la poétique de la négociation
}

\author{
"Mi tótem tiene que ver con ese animal duro de cazar que corre con su propia \\ gracia ». Simanca Pushaina or the poetics of negotiation. \\ "Mi tótem tiene que ver con ese animal duro de cazar que corre con su propia \\ gracia ». Simanca Pushaina o la poética de la negociación.
}

Laura Lema Silva

\section{Introduction}

\begin{abstract}
Entonces te ofrezco La Guajira como asilo, en la ranchería más abandonada, donde se toma agua salitrosa y se come de vez en cuando. [...] Que vivas en carne propia las promesas no cumplidas. Donde no nos importará si hiciste o no hiciste porque no te lo preguntaremos. [...] En esa ranchería no podrán buscarte ni querrán hacerlo porque saben que nosotros no entregamos a quienes aquí se asilan y porque saben que esta sed de vida interminable sólo la podemos soportar los wayuu ${ }^{1}$.

Estercilia Simanca Pushaina, « En las vísperas nacen las cerezas », 2019.
\end{abstract}

La négociation et le dialogue avec les alijunas est une constante dans l'histoire de la communauté indigène wayuu. Les alijunas, ou étrangers, sont littéralement « ceux qui causent la douleur de loin» expression qui fait référence aux armes à feu des colonisateurs et diverses puissances impériales présentes dans le territoire wayuu aux $\mathrm{XVII}^{\mathrm{e}}$ et $\mathrm{XVIII}$ e siècles, lorsque la péninsule de La Guajira devient le champ de bataille politique entre des empires européens en expansion. Pourtant, rapidement, la communauté met en place de complexes relations économiques et politiques basées sur la contrebande avec les alijunas. Elle échange des perles contre des armes qui lui 
permettent de conserver une certaine autonomie politique face aux puissances colonisatrices et évangélisatrices (Acuña J. P., 2005).

2 Des colonisateurs, la communauté wayuu adopte également le bétail, ce qui entraîne de nombreuses modifications dans son organisation économique et sociale tout comme un rapport renouvelé à son territoire et à sa riche tradition orale contenue dans les jayeechis et dans les récits oraux. Ces chants et récits sont porteurs des mythes d'origine et rendent compte du rapport particulier que les wayuu entretiennent avec le temps. En effet, comme le démontre l'anthropologue Weildler Guerra (Guerra W., 2019), la tradition orale, qui est en soi une littérature, décrit les transformations de l'humanité originelle et universelle du wayuu sumaiwa. Ce dernier terme désigne une temporalité transhistorique "clairement sans passé mais tournée vers le futur ». D'après Guerra, l'humanité première se transforme sous l'action des démiurges, en plantes, animaux, vents et montagnes; cette transformation est désignée en wayuunaiki par le mot a'wajanawaa. L'introduction du bétail apparaît, dans la tradition orale, comme le résultat de la transformation de certaines créatures marines qui, à force de sortir de la mer la nuit, auraient été attachées à des arbres et transformées en bovins, moutons et chèvres. Les jayeechis sont ainsi porteurs d'une pensée en constante traduction qui met en évidence les richesses d'une cosmologie caractérisée par un rapport horizontal à l'ensemble des êtres présents dans un territoire qui convoque ses propres cartographies. En effet, W. Guerra démontre que certaines montagnes et pierres constituent la manifestation spatiale du temps transhistorique du mythe et sont la preuve matérielle d'une coexistence entre le monde invisible ou pülasü̈̈ et le monde quotidien des wayuu. L'univers pülasü̈̈ s'introduit constamment dans leur vie quotidienne grâce aux rêves ou aux rituels chamaniques.

3 Les années 1970-1980 marquent un véritable tournant concernant la tradition orale wayuu: les jayeechis et récits oraux sont transcrits en écriture alphabétique conventionnelle et traduits en espagnol ${ }^{2}$ par les intellectuels wayuu Miguel Ángel Jusayú (Jusayú M. A., 1986) et Ramón Paz Iipuana (Paz Iipuana R., 1973) et par l'anthropologue français Michel Perrin (Perrin M. et.al., 1986). Ce phénomène donne naissance à une véritable apogée de la littérature wayuu en écriture alphabétique et en espagnol, dont Estercilia Simanca Pushaina est l'une des principales représentantes. Les années 1970-1980 sont un moment de traduction culturelle profonde, la pensée wayuu est désormais non seulement circonscrite à la littérature orale et à l'écriture pictographique - contenue notamment dans les mochilas wayuu - mais peut également se dire en écriture alphabétique conventionnelle; cette traduction vers l'espagnol s'accompagne d'une véritable transformation culturelle. En plus, entre 1970 et 1990 sont posés les jalons d'une meilleure connaissance de la communauté wayuu par l'État. De nombreux intellectuels et activistes ${ }^{3}$ de la communauté se penchent sur la question de l'auto-reconnaissance des membres de la communauté en tant que wayuu à partir d'un certain nombre de critères qui définiraient leur ethnicité. Ces efforts sont entérinés par la Constitution Politique de 1991 qui reconnaît le caractère multiethnique et pluriculturel de la Nation colombienne, reconnaissance qui est suivie par ce qui, à partir des années 2000, est connu comme le multiculturalisme (Van Cott D.L., 2000). Très rapidement, les politiques multiculturelles vont être dénoncées pour leur caractère d'endiguement de la diversité culturelle, notamment épistémologique, des communautés (Bocarejo D. et Restrepo E., 2011 ; Zambrano M. et Chaves M., 2006). Le principal problème de ces politiques serait qu'elles prolongent les dynamiques 
coloniales en circonscrivant la diversité à un territoire clairement délimité d'un point de vue juridique, c'est-à-dire au territoire ancestral des peuples autochtones. La diversité culturelle se voit réduite au local et acquiert une dimension fortement essentialiste : l'État, par l'intermédiaire du ministère de l'Intérieur, a le dernier mot lorsqu'il s'agit de valider le caractère ethnique d'une communauté donnée.

Dans cette optique, nous pouvons nous demander si les transformations contemporaines des paysages culturels wayuu - transcription et traduction de la tradition orale, usage dominant de l'espagnol dans la littérature contemporaine demeurent vraiment prisonnières de l'exercice du pouvoir étatique par l'intermédiaire des politiques multiculturelles. Pour le dire autrement, ces processus de traduction et ce contact grandissant avec l'État et la société colombienne majoritaire entraînent-t-ils un effacement des particularités épistémologiques wayuu et de la richesse de la pensée mythique de la communauté ? Dans cet article nous nous proposons de faire l'étude de l'œuvre d'Estercilia Simanca Pushaina (Simanca Pushaina E., 2017, 2019) afin de démontrer que la littérature wayuu contemporaine doit être lue au-delà de la constante référence à l'exercice du pouvoir étatique - pensé en termes de domination - sur les communautés indigènes. Avocate de formation, Simanca Pushaina - fille d'un père afro-colombien et d'une mère wayuu - est née en 1975 à Paraíso, la ranchería $a^{5}$ de Mamá Victoria, sa grand-mère, située dans la basse Guajira. La littérature de Simanca est inséparable de son métier. Elle utilise l'écriture comme une arme de défense des droits de sa communauté. «"Manifiesta no saber firmar". Nacido 31 de diciembre » est une nouvelle qui dénonce la discrimination étatique à l'égard des wayuu. Simanca s'est également penchée sur la question de la violence conjugale tout comme sur la crise alimentaire wayuu en dénonçant ainsi les rapports de domination propres à sa communauté. Ce caractère ouvertement politique à l'œuvre dans la littérature de Simanca ne la rend pas pour autant facilement catégorisable. Comme nous le verrons, l'auteure est surtout caractérisée par sa constante transgression des identités: sa position est celle de la négociation; sa production littéraire peut en effet à plusieurs égards être rattachée au rôle de la contrebande économique qui, dans l'histoire de la communauté, permet la conquête d'une certaine autonomie politique par l'intermédiaire de la réinvention culturelle.

5 L'œuvre de Simanca peut par ailleurs être pensée, malgré quelques différences, en lien avec l'apogée actuelle des littératures indigènes des Amériques. À ce propos, il nous semble nécessaire de faire quelques clarifications théoriques. Les littératures autochtones des Amériques sont en lien avec les mouvements sociaux indigènes contemporains et leurs luttes politiques. En ce sens, soulignons que la production esthétique indigène contemporaine ne se limite en aucun cas à la littérature en écriture alphabétique. Miguel Rocha Vivas, spécialiste des littératures des communautés autochtones en Colombie, dont celle de Simanca ${ }^{6}$, explique que la production esthétique indigène peut-être conçue comme une avant-garde puisqu'elle invite à repenser le concept même de littérature (Rocha Vivas M., 2010 et 2016). En effet, il convient de reconnaître l'existence de littératures pictographiques et orales autochtones. Les écrivains et écrivaines indigènes rendent visible leur richesse scripturale - en constante réinvention depuis l'époque coloniale - et font ainsi appel à une prise en compte véritable, dans une optique d'horizontalité, de leurs systèmes de connaissance. De nombreuses productions esthétiques indigènes mettent effectivement en scène un entrecroisement entre l'oralité "traditionnelle» et les écritures pictographiques et alphabétiques ; c'est ce que Rocha Vivas nomme les oralittégraphies. 
La littérature wayuu a des spécificités qui tiennent à son histoire. Contrairement aux oralittégraphies ou à l'oraliture - terme du sénégalais Yoro Fall repris par Elicura Chihuailaf, poète mapuche, pour décrire les littératures indigènes des Amériques - la littérature wayuu contemporaine est alphabétique mais elle ne remplace pas pour autant la littérature orale ni pictographique. Ainsi, les wayuu ont une véritable voix littéraire : Los dolores de una raza d'Antonio Joaquín López (Briscol) est le premier roman d'un membre de la communauté à être publié, il paraît en 1957. Cette voix littéraire, tout comme celle de nombreuses communautés autochtones des Amériques, est en constante réinvention depuis l'époque coloniale, mais a été ouvertement ignorée par les sociétés majoritaires. Approcher cette voix nécessite de se plonger dans l'histoire particulière de chacun des peuples autochtones.

6 Cet article se propose donc, afin de tenter d'être le plus fidèle aux particularités de l'œuvre de Simanca, de partir de l'histoire de la communauté wayuu et de la pensée mythique qui la caractérise. C'est pourquoi nous nous appuierons sur les analyses de l'anthropologue wayuu Weildler Guerra qui, dans Ontología wayuu: categorización, identificación y relaciones de los seres en la sociedad indígena de la península de La Guajira, Colombia, explore les épistémologies wayuu en partant des récits oraux transcrits par Miguel Ángel Jusayú et Ramón Paz Iipuana. Dans cet article, nous ne nous appuierons donc par sur la théorie post ou décoloniale. Cette dernière permet d'attirer l'attention sur les épistémologies occultées par les processus de colonisation et ses métamorphoses, mais elle peine à penser la capacité d'action des sujets autochtones qu'elle conçoit comme subalternes. Dans cette configuration, la production esthétique wayuu serait à comprendre en termes de résistance vis-à-vis de la colonialité du savoir, du pouvoir et de l'être ${ }^{7}$. Elle serait donc en permanence soumise au risque d'être absorbée par un système de connaissance euro-centrique. Sont ignorées ici les stratégies de négociation que déploient les wayuu vis-à-vis des pouvoirs en place tout comme leur propre production de rapports de force politiques. Cela s'accompagne d'une certaine essentialisation des structures épistémologiques indigènes qui sont en réalité, cela est visible lorsqu'on adopte un point de vue historique, en constant mouvement du fait des contacts culturels qui se déploient depuis le début de l'époque coloniale dans la péninsule de La Guajira. Dans cet ordre d'idées, la participation active des wayuu dans les réseaux de contrebande économique leur donne une marge de manœuvre certaine qui se traduit dans leur capacité d'interlocution active, pacifique ou violente, avec l'extérieur. Nous démontrerons que, dans la littérature de Simanca, l'usage de l'écriture alphabétique peut s'assimiler à une forme de contrebande qui permet de rendre visible le caractère mouvant de l'identité wayuu et des systèmes de pensée qui la nourrissent.

7 Parler de réinvention culturelle en lien avec la contrebande et situer l'œuvre d'Estercilia Simanca dans ce registre, signifie la penser au-delà de l'exercice de la domination des manifestations majoritaires de l'État ${ }^{8}$ qui seraient marquées par la colonialité. Nous pouvons en effet poser la question du contact avec l'État autrement, en nous demandant en quoi étudier les processus de traduction à l'œuvre dans la production littéraire de Simanca revient à traiter des innovations créatrices, de la capacité d'action de l'auteure et de la communauté wayuu en général. Répondre à cette question signifie penser le potentiel émancipateur de sa littérature et comprendre les relations qu'entretient la communauté wayuu avec l'extérieur. Nous verrons que les nouvelles de Simanca ont un caractère clairement politique qui met en évidence la multiplicité et la complexité des modes de vie des wayuu, en particulier des femmes, 
tout en transmettant la profondeur de la pensée mythique de ce peuple indigène. Ainsi, nous nous pencherons sur ce que signifie, chez Simanca, se nommer, nommer les femmes wayuu et nommer l'État qui opprime la communauté. Nous verrons que nommer revient à rendre compte des rapports complexes qui se jouent, chez les wayuu, entre le dedans et le dehors de la communauté.

8 Ainsi, nous traiterons dans une première partie des stratégies des wayuu qui, malgré le fait d'être immergés dans des relations de pouvoir, arrivent à récupérer des espaces de négociation politique, à penser et à nommer l'État. Cela nous permettra ensuite de nous pencher sur la condition multiple des femmes wayuu qui révèle à la fois la rigidité des structures sociales de la communauté tout comme les stratégies utilisées par elles, lorsqu'elles le peuvent, pour les mettre en question. Dans un dernier point, nous étudierons le nom que l'auteure s'attribue au fil de ses nouvelles pour saisir la dimension politique des femmes Irama.

\title{
I. Mettre en avant la voix wayuu qui pense et nomme l'État
}

\author{
Ese día me enteré de que mi tío Tanko Pushaina se llamaba Tarzán, que Shankarit se \\ llama Máximo, que Jutpunachón se llamaba Priscila, que Yaya se llamaba Clara, que \\ Castorila se llamaba Cosita Rica, que Kawalashiyú se llamaba Marquesa, que \\ Anawachón se llamaba John F. Kennedy, que Ashaneish se llamaba Cabeza [...] que el \\ primo Rafael Pushaina se llamaba Raspahierro, que mi primo Matto se llamaba \\ Bolsillo, y por un momento temí que conmigo pasara lo mismo?. \\ Estercilia Simanca Pushaina, " "Manifiesta no saber firmar". Nacido 31 de diciembre \\ $», 2005$.
}

9 À travers " "Manifiesta no saber firmar". Nacido 31 de diciembre », Estercilia Simanca rend compte d'un univers wayuu où l'alijuna s'invite de manière arbitraire et profite de l'hospitalité de la communauté. Simanca décrit l'intrusion des étrangers qui imposent leurs intérêts électoraux avec des fausses promesses comme, par exemple, la construction des infrastructures nécessaires pour garantir l'accès à l'eau, bien rare dans la péninsule de La Guajira. Ainsi, apparaissent plusieurs personnages que la jeune narratrice de la nouvelle, Coleima Pushaina, nomme "Candidat ", " Gouverneur » ou encore "Maire » et qui sont rendus responsables, avec la Registraduría Nacional del Estado Civil, institution qui s'occupe de l'attribution des cartes d'identité aux citoyens colombiens, de l'attribution des noms insultants aux wayuu de tous âges : les enfants se voient également attribuer des documents d'identité pour qu'ils puissent aller voter pour l'un des «Candidats» qui, une fois élu, oublie les promesses faites pendant la période électorale. Ainsi, dans les documents officiels de l'État, tous les wayuu seraient nés le 31 décembre :

[...] me bautizaron el 5 de septiembre de 1970 y mi fecha de nacimiento 31 de diciembre de 1965. Decía que yo no era hija de Korouna Pushaina ni de Colenshi Jusayú, sino de María Santa Pushaina con Domingo Santo Jusayú, y que yo no me llamaba Coleima Pushaina, sino Faride Abuchaibe; que todos los niñitos bautizados ese día se llamaban Faride y Eduardo Abuchaibe. Ahora entiendo por qué todos me dicen la turca ${ }^{10}$.

10 Comme le démontre la citation, deux formes d'exercice du pouvoir sont en jeu dans l'attribution des noms insultants aux membres du peuple wayuu. La première concerne l'imposition d'une identité. L'appartenance à un système de parenté spécifique à la communauté est ouvertement ignorée par l'État et ses fonctionnaires. Simanca évoque 
l'effacement du clan Pushaina et du clan Jusayú au profit du nom Abuchaibe. Dans la culture wayuu, les clans symbolisent l'appartenance à une humanité originelle et universelle; ils sont en plus garants de la reproduction d'une organisation sociale et politique matrilinéaire. Par ailleurs, chacun des e'irrukü, ou clans, est rattaché à un lieu d'origine - ii en wayuunaiki - qui symbolise l'appartenance des wayuu contemporains au wayuu sumaiwa, rattachement qui, d'après Weildler Guerra, garantit la coexistence pacifique des membres de la communauté. Attribuer un nom différent aux wayuu revient donc à ignorer leur organisation sociale et politique, ainsi que leur rapport au territoire, c'est donc porter atteinte à la reproduction d'un système socio-politique complexe.

11 Nommer les wayuu et leur attribuer une même date de naissance est aussi un mécanisme pour leur imposer un rapport au temps qui est celui du calendrier occidental. Le 31 décembre est une date qui nous situe immédiatement dans l'entre deux : aux yeux de l'État, l'existence même des wayuu est suspendue dans un moment de transition. Dans cet ordre d'idées, l'entreprise de l'État consiste non seulement à nommer les wayuu selon ses propres normes alphabétiques - dans leurs documents d'identité, les fonctionnaires indiquent que les wayuu ne savent pas signer, en ignorant leur rapport à l'écriture pictographique - mais aussi à leur imposer une temporalité autre. Simanca dénonce donc la volonté étatique d'instaurer un contrôle de la population indigène au service de fraudes électorales. Fixer l'existence wayuu un 31 décembre revient à la suspendre dans le temps et révèle le rapport ambivalent qu'entretiennent les fonctionnaires et les candidats avec la communauté. En effet, les candidats séduisent la communauté, pour la décevoir une fois la période électorale passée. Cette situation répétitive dégage une première impression chez le lecteur: la communauté wayuu peut difficilement récupérer des espaces d'action politique, la domination des signes majoritaires de l'État - symbolisés par l'écriture alphabétique et par l'espagnol - tout comme la bureaucratie étatique semblent à première vue insurmontables. Néanmoins, par l'intermédiaire de l'écriture, Simanca réussit à mettre en évidence les marges d'action des wayuu.

Comme nous l'avons souligné dans l'introduction, l'auteure est l'une des écrivaines qui participe le plus activement à l'expansion de la littérature wayuu suite aux processus de traduction et de transcription des années 1970-1980. À première vue, sa littérature s'inscrirait donc dans la problématique qu'elle dénonce, c'est-à-dire celle du pouvoir exercé par l'écriture alphabétique et par l'espagnol envers une communauté caractérisée par d'autres formes d'écriture et par une riche tradition orale. Ce paradoxe doit cependant être lu à la lumière de l'histoire. Il est en effet comparable à la place de la contrebande économique dans les stratégies déployées par la communauté pour garder son autonomie politique. Ainsi l'usage de l'écriture alphabétique et de l'espagnol apparaissent comme des armes de négociation; il s'agit de faire de la contrebande avec l'écriture pour mettre à nu le caractère superficiel des structures étatiques et du système électoral qui le nourrit. L'écriture de Simanca permet de rendre visible non pas une situation d'oppression mais plutôt la capacité des wayuu à exprimer leurs points de vue. Faire l'usage de l'écriture alphabétique et de l'espagnol revient dans " "Manifiesta no saber firmar" " à nommer l'État et à dévoiler ses stratégies. En effet, dans sa dernière nouvelle, à caractère autobiographique, "En las vísperas nacen las cerezas ", Estercilia Simanca Pushaina revient sur la question de la naissance des wayuu le 31 décembre : 
De tanto aceptar sin saber qué se nos preguntaba, terminamos celebrando de una manera distinta el fin de año, porque un día cualquiera todos «Nacimos un 31 de diciembre». Al Estado se le olvidó el nombre de las otras lunas y quiso que como celebración de fin de año también festejáramos nuestro cumpleaños ${ }^{11}$.

Dans cette citation, il est intéressant de noter que la naissance des wayuu le 31 décembre est attribuée à un oubli de la part de l'État. En faisant allusion à « el nombre de las otras lunas ", Estercilia Simanca fait référence au temps wayuu, inconnu par l'État. L'auteure pointe ainsi du doigt l'analphabétisme étatique envers la richesse épistémologique de cette communauté. Un renversement est donc en scène ${ }^{12}$, l'État ignore les formes d'écriture et le rapport au temps d'un peuple faussement considéré " analphabète ». Cette méconnaissance va également révéler la superficialité des temps bureaucratiques de l'État, des stratégies du système électoral colombien et des masques utilisés par les personnes au pouvoir. Estercilia Simanca dénonce certes l'exercice du pouvoir qui trouve son paroxysme dans l'attribution de noms insultants aux wayuu, mais n'hésite pas à récupérer cette pratique et à l'utiliser contre l'État qu'elle nomme et dont elle dévoile une superficialité confuse aux yeux des wayuu :

Esa vez llevaron unos papeles grandotes que tenían la imagen de ese hombre que se llama «Candidato». Ellos tienen nombres extraños por lo que nada de raro tendría que ese señor se llamara así [...] Hay otra casa que se llama Alcaldía y el que vive ahí se llama Alcalde, pero al principio también se llamó igual que el otro... Candidato. ¿No saben ellos que tantos nombres pueden causar confusión? ${ }^{13}$

Cette dénonciation de la superficialité des temps bureaucratiques et étatiques est encore plus parlante lorsque l'on sait que la narratrice de la nouvelle est une jeune fille. Son regard innocent est finalement un moyen de dénoncer avec plus de force la confusion propre au fonctionnement étatique. Le temps wayuu qui dans la citation précédente est associé à la lune et donc à la nature apparaît comme allant de soi. En plus de mettre en évidence ces contrastes, Simanca, en utilisant l'écriture alphabétique et l'espagnol, convoque un lecteur actif et connaisseur de la culture wayuu et de ses mythes. En effet, dans la citation, la jeune narratrice associe les maisons des fonctionnaires de l'État avec leur nom. Le "Candidat" se transforme en "Maire " lorsqu'il est à la mairie ce qui entraîne un changement de sa personnalité intrinsèque. Dans son étude du statut ontologique des plantes, des animaux et de certains objets importants dans la vie quotidienne de la communauté, Weildler Guerra (Ibid., p. 96) explique que l'un des éléments qui prouve que pour les wayuu l'ensemble des êtres partageant le territoire ont un statut ontologique de personne est que leur corps est conçu comme leur maison. Ce rapport avec les êtres qui habitent le territoire serait proche de ce que Eduardo Viveiros de Castro nomme le «perspectivisme » (Viveiros de Castro E., 2010) : pour les wayuu, le corps est semblable à un habit qu'adoptent les êtres venus du temps transhistorique et qui cache la condition humaine universelle que tous les êtres partagent dans cette temporalité parallèle. En donnant la parole à une jeune fille, et en mettant en avant sa propre perception des étrangers, Simanca nous met face à la conception du monde des wayuu et révèle donc toute la complexité de leurs structures de pensée. Nous pouvons dès lors avancer qu'en convoquant un lecteur actif Simanca invite au dialogue avec ceux qui sont extérieurs à la communauté. Un dialogue qui est en plus essentiel dans une culture qui accorde une place centrale au pouvoir de la parole dans la résolution des conflits (Guerra W., 2002). En effet, la naïveté des wayuu vis-à-vis des candidats aux élections est surtout révélatrice d'une confiance en la parole 
de l'autre et en ses promesses. Avec cette nouvelle, Estercilia Simanca laisse ouverte la porte du dialogue avec l'État et la société colombienne.

" "Manifiesta no saber firmar" ", problématise les relations entre les wayuu et les alijunas sans les réduire à un schéma dichotomique en termes de dominants et de dominés. La dénonciation de l'exercice du pouvoir étatique qui à première vue semble accabler la communauté wayuu ne se formule cependant pas en faisant d'elle une victime. Estercilia Simanca met en avant la voix de la jeune narratrice wayuu et démontre que nommer l'État et ses fonctionnaires revient à révéler leur caractère superficiel et à exposer le complexe rapport à la vie et au temps propre à la communauté wayuu. Le caractère de dénonciation n'exclut pas la volonté de dialogue qui se révèle lorsque la jeune narratrice nomme les fonctionnaires dans une tentative de compréhension de leurs nombreux visages. Ainsi, nous sommes face à une littérature qui pense les contacts de la communauté avec l'extérieur en récupérant la capacité d'action des wayuu dans une situation d'exercice du pouvoir politique. Cela caractérise également la critique effectuée par l'auteure dans son examen de la condition des femmes wayuu où elle rend compte de positions sociales multiples en démontrant qu'elles ne sont pas forcément immuables et en révélant par là même des éléments fondateurs de la cosmologie et des structures matrimoniales wayuu.

\title{
II. Les femmes wayuu : des conditions sociales multiples
}

\begin{abstract}
Esa transgresión tiene que venir de alguien del mismo pueblo, no de que yo fui a Madrid y vine con unas ideas liberadoras para mi pueblo. Soy transgresora por el hecho de ser wayuu, bueno que la mujer wayuu debe tener más libertades sí, pero la mujer wayuu [ya] tiene libertades y las va adquiriendo mientras va adquiriendo independencia económica como ocurre también en Occidente y cuando están en Europa hablando de movimientos feministas de los 80 de los 70, de los 60, en la comunidad wayuu ya existían mujeres Julamias [... $]^{14}$.

Entretien avec Estercilia Simanca Pushaina, Riohacha, 29 juin 2017.
\end{abstract}

La communauté wayuu est une société matrilinéaire et patriarcale. Les femmes sont associées à la terre, Mma, et les hommes à la pluie, Juyá : les hommes comme la pluie sont mobiles, les femmes, elles, sont pensées en lien avec le caractère permanent de la terre, cela explique la pratique exclusive de la polygamie masculine. La littérature d'Estercilia Simanca a souvent été lue comme féministe, néanmoins l'auteure s'oppose à ce qualificatif et explique que sa littérature est plutôt transgressive, puisqu'elle parlerait non pas depuis les problématiques abordées dans les universités occidentales mais depuis la réalité de sa communauté. Le caractère transgressif de la littérature de Simanca est lié à l'énonciation d'une critique non seulement à l'égard des relations des fonctionnaires d'État vis-à-vis des wayuu mais concerne aussi les structures de pouvoir propres à sa communauté. Il importe de préciser que les cadres d'analyse concernant cette posture que l'auteure adopte ne doivent pas ignorer les particularités de la culture wayuu, d'où la difficulté de lire l'œuvre de Simanca à partir des études féministes ou intersectionnelles. Dans sa littérature, plus qu'adopter un discours féministe, Simanca rend visibles les multiples conditions des femmes de sa communauté, et par là même elle dévoile les structures de pouvoir et de connaissance qui fondent un système matrimonial qui peut se traduire par l'exercice de la violence conjugale. 
17 Au fil de ses nouvelles, Estercilia Simanca fait le portrait de nombreuses femmes dont les surnoms symbolisent une condition sociale et une autonomie différentes vis-à-vis des rôles traditionnels de genre dans la communauté. Les princesses wayuu sont celles qui vivent le rituel de l'enfermement après l'arrivée de leurs règles. Ce rituel, décrit dans «Encierro de una pequeña doncella », les prépare à leur vie de femmes mariées. Elles apprennent notamment à tisser l'écriture pictographique propre à la communauté. Lorsqu'Estercilia Simanca rend compte de la pratique de l'enfermement des jeunes filles, elle démontre l'enracinement de cette pratique dans le temps transhistorique du mythe ou univers pülasüü :

Iiwa duró un año soñando con la doncella desconocida que le revelaba con sus manos y sin pronunciar una sola palabra, más y más secretos del tejido wayuu. [...] En el último sueño con la doncella desconocida, porque nunca los volvió a tener, Iiwa recordó la leyenda de Waleker y descubrió que aquella doncella era la misma que se había convertido en araña al ser descubierta por su protector. El cazador que la salvó al encontrarla sola y desamparada en el monte la adoptó y la llevó a su ranchería y, en agradecimiento, todas las noches, cuando nadie la veía, la doncella desconocida halaba hilos de su boca y realizaba hermosos tejidos para el cazador. Una noche vista por él y al ser sorprendida se convirtió en araña y huyó hacia un árbol. Desde entonces quedó convertida en Waleker, en araña ${ }^{15}$.

Ici, le lecteur plonge dans l'univers pülasüü de la communauté. Pour les wayuu, Iiwa, est une étoile - masculine dans leur cosmologie - pensée comme la sœur de la pluie, Juyá. Elle est donc associée, d'après Weildler Guerra Curvelo (Ibid., p. 87), à la fertilité. Par ailleurs, la transformation en araignée de la jeune fille qui apprend à Iiwa à tisser est semblable à la transformation de l'humanité originelle wayuu en plantes, animaux et montagnes. Dans la cosmologie wayuu, c'est la plante de coton qui aurait transmis la connaissance associée au tissage à l'araignée Waleker qui ensuite la transmet aux êtres humains. D'après Guerra Curvelo, cet apprentissage fonde, chez les wayuu, une conception de la culture non pas comme construction humaine mais comme étant liée à la transmission grâce, notamment, au rôle des plantes, symboles de sagesse et de connaissance. Cet extrait, en plus de plonger le lecteur non wayuu dans les univers oniriques propres à la communauté, met en scène la variabilité du mythe. Ceci démontre que l'usage de l'espagnol et de l'écriture alphabétique conventionnelle n'est en aucun cas un obstacle pour l'introduction des spécificités épistémologiques wayuu qui sont réactualisées dans l'écriture alphabétique de Simanca. De plus, cet extrait révèle la position complexe de l'auteure vis-à-vis de l'enfermement. Ce dernier prépare certes les jeunes filles à leur vie de mariées mais il s'agit aussi d'un moment d'apprentissage de l'ensemble de la connaissance associée au monde pülasüü. Les femmes sont en ce sens les gardiennes de la connaissance venue du wayuu sumaiwa. L'apprentissage transmis par Waleker, n'est pas uniquement utilitaire, comme il pourrait être à première vue perçu par un lecteur non wayuu, il pointe au contraire du doigt notre propre analphabétisme et incapacité à lire l'écriture pictographique (Rocha Vivas M., 2016). Une écriture qui marque l'introduction du temps transhistorique du mythe dans la vie quotidienne des wayuu. Soulignons donc que nous ne sommes pas ici dans une optique d'identification des jeunes filles wayuu qui vivent le rituel de l'enfermement à une nature essentialisée. La cosmologie wayuu se base sur une transmission de la connaissance par les plantes et les animaux qui, rappelons-le, ont un statut ontologique de personnes dotées d'une intentionnalité et d'un code moral. L'art du tissage est aussi celui de la littérature pictographique, ce sont les femmes qui possèdent ce savoir et cela leur donne un pouvoir indéniable dans le système socio- 
politique wayuu. Sans cette connaissance le devenir politique de la communauté serait compromis, puisque cette écriture pictographique est une forme de communication directe avec le monde pülasüü. Sans elle, la guérison des maladies ou la résolution des conflits à l'intérieur et entre les clans serait impossible.

19 Face à la question des mariages forcés, la posture de Simanca est celle de la dénonciation. "Bultito llorón. Cara de indio" est une nouvelle qui met à nu la condition des femmes obligées à se marier avec des hommes âgés et à subir leur violence. Estercilia s'oppose avec virulence à ces unions et à l'inaction dans laquelle se retrouvent les femmes qui les subissent:

Mientras cuelgo un chinchorro en la enramada para que mamá descanse, ella carga mi bultito llorón, me dice que esperará hasta que el ombligo se le caiga para llevárselo y enterrarlo en nuestra ranchería, quisiera que se lo llevara a él también y si quisiera lo enterrara al pie del árbol de cerezo. Mamá toca mi vientre y dice que soy muy fértil, no solo por parir este bultito que llora sino porque los animales que dieron por mí parieron antes del año, mamá sólo vino a cobrar la sangre que derramé en el parto ${ }^{16}$.

20 La narratrice de la nouvelle, mère d'un enfant non désiré, est condamnée par un système social polygame qui ne protège pas les femmes des violences conjugales. Dans la citation, est évoquée la pratique du bridewealth qui se différencie, comme l'explique l'anthropologue Alessandro Mancuso, de la dot dans le sens où c'est la famille de l'époux qui « paye » un mariage ou la naissance d'un enfant (Mancuso, A., 2020). Cette pratique doit être comprise en lien avec le kojutü, notion qui en wayuunaiki désigne la valeur inhérente à toute personne. Le kojutü fonde un système de compensation caractérisé par la réciprocité entre personnes et familles. Dans le cas spécifique du mariage, le bétail et les colliers fabriqués à partir des tuumas ${ }^{17}$ constituent les biens de compensation matériels que la famille de l'époux donne en échange des biens de la mariée et de sa valeur liée à la perpétuation du lignage, la société wayuu étant matrilinéaire. Ainsi, insistons sur le fait que Simanca ne dénonce pas le bridewealth mais les violences envers les femmes qui sont une réalité chez certaines familles wayuu. La jeune narratrice de «Bultito llorón. Cara de indio » est abandonnée à son propre sort et trouve comme unique solution de fuir dans ce qu'elle nomme le «village » des alijunas. Ainsi, Simanca rend visibles les violences qui condamnent ces femmes, invisibles et sans nom à l'inaction. En ce sens, Simanca dévoile les mécanismes d'un système matrimonial qui ne protège pas les femmes victimes de violences conjugales.

21 Pour revenir sur la notion de « valeur » dans la commuanuté wayuu, soulignons que dans sa nouvelle « Julamia » Simanca rend compte d'un autre aspect de la condition féminine dans la communauté et révèle par là même la forte hiérarchisation sociale existant chez les wayuu. En effet, les hommes choisissent généralement de se marier avec plusieurs femmes plutôt qu'avec une femme riche ou Julamia, dont la compensation à payer serait très élevée. Dans cette configuration spécifique ces dernières peuvent partir avec leur fortune et choisir un mari :

La Julamia vio que la inmensidad de su fortuna era tan grande como la palabra amor. Muchos hombres la esperaban, ahora sí la visitaban [...]. Era dueña de sus pasos ya a su esposo lo buscaría en otros territorios, haría una carrera de caballos y sería ella quién dotaría al mejor y más joven de los jinetes ( Julamia », 141-156) ${ }^{18}$.

22 Ainsi, insistons là-dessus, le caractère transgressif de la littérature de Simanca ne peut pas être lu comme une posture féministe. Là où il intervient, c'est dans la mise en évidence du fonctionnement social de la communauté wayuu et des structures de 
pensée qui le fondent. Simanca rend compte d'une société complexe, fortement hiérarchisée d'un point de vue économique et productrice de rapports de pouvoir. En un sens, la dimension transgressive de sa littérature repose sur le fait qu'elle s'éloigne de toute conception essentialisée et réductionniste de la culture wayuu.

En décrivant la quotidienneté de la vie de ces trois femmes, l'auteure dévoile le fonctionnement $\mathrm{du}$ système socio-politique wayuu. Simanca fait le portrait de différents vécus et montre que la position des femmes dans cette société polygame et patriarcale n'est pas univoque. Pour les femmes invisibilisées par les violences conjugales, le monde alijuna réapparaît. Contrairement à ce que nous avons exposé concernant " "Manifiesta no saber firmar" ", l'alijuna ne fait pas irruption dans l'univers wayuu sans être invité, cette fois-ci il est recherché activement par la protagoniste de "Bultito llorón. Cara de indio» qui voit dans les "maisons" des alijunas un espace de conquête d'un devenir plus libre. Cette illusion est rapidement déçue lorsque la narratrice réalise que vivre chez les alijunas signifie subir la discrimination et le racisme de la société majoritaire. C'est ce dernier point qui amène Estercilia Simanca à proposer des alternatives pour penser la place des wayuu et, d'un point de vue plus général, de la diversité culturelle indigène, dans la société colombienne. Cela est en lien avec le nom que l'auteure s'attribue, reflet de sa position entre l'intérieur et l'extérieur de la communauté.

\title{
III. Se nommer : écrire pour le monde en réactualisant la « tradition » wayuu
}

\begin{abstract}
Mi animal totémico no tiene nada que ver con el clan que está consignado en mi partida de nacimiento [...]. Mi tótem tiene que ver con ese animal duro de cazar, que corre con su propia gracia, muchas veces es cazado y su carne dicen que es exquisita, nunca lo he probado y dudo que lo haga, soy como mi abuelo que nunca comió su propia carne, el cerdo o zaino, « No puedo comerme a mí mismo » me dijo. Me han herido muchas veces, pero he sabido cicatrizar con mi propia saliva las heridas de ese cervatillo que soy yo misma... Sí, soy Irama ${ }^{19}$.

Estercilia Simanca Pushaina, « En las vísperas nacen las cerezas », 2019.
\end{abstract}

Dans la production littéraire de Simanca nommer ne veut pas dire fixer une identité ou essentialiser la manière d'être au monde des membres de la communauté wayuu. Nommer c'est plutôt montrer la multiplicité qui traverse ce peuple indigène, qui fait de lui une société difficilement réductible à une quelconque vision préconstruite de ce qu'est être indigène. Simanca rend compte d'une société dans laquelle parfois fait violemment irruption l'étranger ou alijuna, qui d'autres fois est recherché activement par les wayuu. C'est dans cette interaction entre l'intérieur et l'extérieur de la communauté que se construit la figure d'une dernière femme qui prend vie dans les nouvelles de Simanca, la femme Irama :

Mi nombre es Mireya. Así me llamó una madrina árabe que nunca más volví a ver, pero todos me dicen Irama a mis espaldas, no me molesta porque lo soy. Por no pasar por el encierro mi espíritu de niña quedó prendido en mí, no sé cocinar y no voy a aprender, ya no me gusta llevar el cabello largo y mis joyas están mejor en la múcura que en mi garganta, no camino detrás de mis hermanos sino al lado de ellos, no sé guardar silencio, pero sí los secretos, estos se van siempre conmigo ${ }^{20}$.

Irama est une femme rebelle qui, d'après la citation, évolue au même niveau que les hommes de la communauté. Son pouvoir devient manifeste notamment dans 
l'interaction avec la société majoritaire. Estercilia Simanca, puisqu'elle n'a pas vécu le rituel de l'enfermement, se nomme elle-même Irama :

Yo soy una mujer Irama, yo no pasé por el encierro. Cuando una mujer wayuu no pasa por el encierro es considerada un venado, o sea eternamente infantiles y rebeldes, una mujer Irama, ahí, loquita ${ }^{21}$.

D’après Simanca, sa position dans la communauté n'est pas celle de la préservation d'une quelconque identité indigène. Elle a une certaine liberté d'action qui s'accompagne néanmoins d'une grande responsabilité, celle de faire entendre la voix des membres de sa communauté et de rendre visibles, en Colombie et dans le monde, les problèmes politiques qui la traversent, c'est-à-dire les abus de l'État et des multinationales ${ }^{22}$. Le caractère politique de la littérature de Simanca est donc à penser à partir du mélange entre son appartenance aux univers culturels wayuu, occidental et afro-colombien ${ }^{23}$. L'écriture alphabétique apparaît ici comme la marque de ce mélange culturel qui amène l'auteure à s'éloigner de tout discours qui essentialise les communautés indigènes ou qui les rend victimes d'un quelconque système de domination :

Hoy como nunca he recordado cuando [mi abuela] me dijo « Que bonito cuando el papel habla contigo » (leer). Ella no me habló de la Madre Tierra, me habló de territorio, porque a dónde fuere no soy más que una pequeña extensión de mi cordón umbilical enterrado al pie del árbol de cereza en la ranchería de mis abuelos. No me enseñó a hablar con la luna ni con el sol; solo por la certeza que cada lluvia cumplo años y cada luna pago impuestos ${ }^{24}$.

Il est intéressant de noter ici l'évocation de la Mère Nature et la distance établie avec la notion de territoire, ainsi que la mise en avant du temps wayuu - pluies et lunes - et sa mise en contraste avec le fait de parler avec la lune et le soleil. Ce qu'Estercilia Simanca vise ici, c'est le discours et les prises de position de nombreux intellectuels et écrivains indigènes et non-indigènes proches de celui que l'on retrouve dans la pensée décoloniale dans ses prises de position sur l'Abya Yala ${ }^{25}$. Lorsque Simanca écrit «la certeza que cada lluvia cumplo años y cada luna pago impuestos » elle se situe immédiatement à l'intérieur des temporalités occidentale et wayuu. Le fait de " parler avec le papier ", en plus de confirmer son appartenance à ces univers multiples, constitue également une façon de construire des ponts et de mettre en place des dialogues, dans ce cas précis, le papier et l'écriture dialoguent avec le monde wayuu, ce qui se traduit par un apprentissage mutuel. La position d'Estercilia Simanca correspond à celle, active, des wayuu dans leur conception d'un extérieur que parfois ils invitent et recherchent. Ainsi, dans ce renouvellement récent des univers culturels, les wayuu occupent une position d'action politique qui est très proche de celle qu'ils adoptent concernant la contrebande économique. En effet, l'on pourrait dire que la dimension émancipatrice de la littérature de Simanca réside dans sa conscience concernant l'histoire de sa communauté et les modifications culturelles qui la traversent. Lorsque, dans notre entretien du 29 juin 2017, l'on évoque la question de la contrebande, Simanca m'explique : « ¿en qué momento el blanco lo etiqueta como contrabando? cuando lo de nosotros es simplemente un intercambio y es un diálogo de Occidente con el pueblo wayuu, y el pueblo wayuu toma de Occidente lo que le sirve, lo que le conviene $»^{26}$. Nous pouvons par conséquent avancer que l'objectif de Simanca est de faire de sa littérature un instrument de médiation. L'usage de l'écriture est, en ce sens, une manière de tenter de rééquilibrer les capacités différentielles de pouvoir existantes entre les wayuu et la société colombienne majoritaire. 
De manière similaire au phénomène de la contrebande économique des $\mathrm{XVII}^{\mathrm{e}}$ et $\mathrm{XVIII}$ siècles, les écrivains et écrivaines wayuu contemporains adoptent l'écriture alphabétique et l'espagnol comme un moyen de faire entendre leurs voix. Sa condition de femme Irama permet à Estercilia Simanca de devenir la porte-parole des problématiques qui traversent sa communauté non seulement en Colombie mais aussi dans des circuits transnationaux. En effet, la contrebande littéraire à l'œuvre dans les nouvelles de Simanca prend une ampleur supplémentaire lorsque l'on pense aux circuits transaméricains d'écrivains indigènes qui ont donné naissance à de nouvelles formes de cosmopolitisme et à de nouveaux centres littéraires. Dans la droite ligne de cet entre-deux qu'elle habite, Simanca explique que l'usage de l'espagnol dans sa littérature la rend latino-américaine dans le sens où elle peut entrer en dialogue avec d'autres communautés :

[Ser latinoamericano es] ser más universal pero en idioma español, llevar la realidad del pueblo al que perteneces a todos los hablantes hispanos, es eso, y que alguien de Guatemala te diga, "ay eso pasa en mi comunidad también" y necesariamente no tiene que ser indígena el que te lo diga ${ }^{27}$.

Cette appartenance au mondial (Duchesne J., 2015) et la visée cosmopolite de la littérature d'Estercilia Simanca ne se font pas pour autant au détriment de la diversité de la communauté wayuu. Simanca peut prétendre à un discours sur l'universel uniquement parce qu'elle est profondément enracinée dans sa réalité locale. En effet, cet enracinement lui permet de repenser le statut de la diversité culturelle en Colombie en l'émancipant du local pour lui donner un caractère plus universel. Le monde peut ainsi apprendre des wayuu et de la richesse de leur pensée. De manière similaire à l'introduction du bétail dans l'ensemble mythique wayuu pendant la colonisation, la littérature d'Estercilia Simanca est la preuve vivante de la réinvention culturelle de sa communauté. Une réinvention qui n'entraîne pas une perte de ses spécificités épistémologiques mais qui est plutôt une occasion de démontrer la porosité de ces systèmes de pensée qui désormais dialoguent avec le monde. C'est là que réside le potentiel émancipateur de cette poétique de la négociation.

\section{Conclusion}

Estercilia Simanca Pushaina met en scène, dans sa production littéraire, une identité wayuu mouvante, élément qui témoigne de la centralité de la réinvention culturelle de la communauté lorsque l'on souhaite penser sa capacité d'action politique. Ses nouvelles cherchent à nommer les membres multiples de la communauté et révèlent ainsi la richesse de l'organisation politique et sociale des wayuu tout comme ses limites. Simanca met en outre en avant la coexistence entre le temps quotidien de la communauté et le temps transhistorique de l'univers pülasüü. Ainsi, Estercilia Simanca fait à la fois le portrait de la quotidienneté des wayuu et convoque un lecteur actif capable de comprendre la profondeur de la pensée de la communauté.

La littérature de Simanca Pushaina est une littérature d'interstices, elle problématise les espaces de contact entre les cultures en prouvant qu'ils sont aussi des opportunités de création. En effet, le rapport au temps des wayuu est traduit, dans sa profondeur, dans l'écriture alphabétique. L'usage de l'espagnol répond à la volonté de l'auteure d'inscrire sa littérature dans des circuits qui dépassent ceux de sa communauté et même ceux de la Nation colombienne. Estercilia Simanca produit une littérature latino- 
américaine qui, dans ses propres termes, n'a pas de juridiction et possède une vocation à dialoguer avec le monde en s'inscrivant dans la réalité des wayuu. Une réalité qu'elle dépeint dans sa complexité en renonçant à toute forme d'essentialisme.

\section{BIBLIOGRAPHIE}

Acuña, José Polo, Etnicidad, conflicto social y cultura fronteriza en la Guajira (1700-1850), Bogota, Ediciones Uniandes, 2005.

Bocarejo, Diana et Restrepo, Eduardo (éd.), Revista colombiana de antropología, ICANH, Volumen 47 (2), julio-diciembre 2011.

Capucine et Hurtado López Fatima, Cahier des Amériques Latines: Philosophie de la libération et tournant décolonial, numéro 62, 2009.

Chaves, Margarita et Zambrano, Marta, « From blanqueamiento to reindigenización: Paradoxes of mestizaje and multiculturalism in contemporary Colombia ", in European Review of Latin American and Caribean Studies, numéro 80, Avril 2006, p. 5-23.

Deleuze, Gilles et Guattari, Félix, L'anti-CEdipe. Capitalisme et schizophrénie, Paris, Les Éditions de Minuit, 1972.

Duchesne Winter, Juan, Hermosos invisibles que nos protegen. Antología wayuu, Pittsburgh, Instituto Internacional de Literatura Iberoamericana, 2015.

Guerra Curvelo, Weildler, La disputa y la palabra. La ley en la sociedad wayuu, Bogota, Ministerio de Cultura, 2002.

Guerra Curvelo, Weildler, Ontología wayuu: categorización, identificación y relaciones de los seres en la sociedad indígena de la península de La Guajira, Colombia, (Thèse en Anthropologie), Universidad de los Andes, 23 avril 2019.

Guerra Curvelo Weildler, «El otro Isaacs », Revista entornos, 14 juin 2019. Disponible sur : http:// revistaentornos.com/el-otro-isaacs/. Consulté le 8 juin 2020.

Jusayú, Miguel Ángel, Achi'ki. Relatos Guajiros, Caracas, Universidad Católica Andrés Bello-Centro de Lenguas Indígenas, 1986.

Laurent, Virginie, Comunidades indígenas, espacios políticos y movilización electoras en Colombia, 1990-1998. Motivaciones, campos de acción e impactos, Bogota, IFEA, ICANH, 2005.

Mancuso, Alessandro, « The place of livestock in human-non-human relationship among the Wayuu », Amerindia Socio-cosmologies between the Andes, Amazonia and Mesoamerica, Hallbmayer, Ernst (éditeur), New York, Routledge, 2020, p. 306-333.

Paz Iipuana, Ramón (1972), Mitos, leyendas y cuentos guajiros, Caracas, Instituo Agrario, 1973.

Perrin, Michel, et al., Folk Literature of the Goajiro Indians, 2 vol., Los Angeles, University of California-UCLA Latin American Center Publications, 1986.

Rocha Vivas, Miguel, Palabras mayores, palabras vivas. Tradiciones mítico-literarias y escritores indígenas en Colombia, Bogota, Fundación Gilberto Alzate Avendaño, 2010. 
Rocha Vivas, Miguel, Mingas de la palabra. Textualidades oralitegráficas y visiones de cabeza en las oraliteraturas y literaturas indígenas contemporáneas, La Havane, Fondo Editorial Casa de las Américas, 2016.

Sibertin Blanc, Guillaume, Politique et État chez Deleuze et Guattari. Essai sur le matérialisme historicomachinique, Paris, Presses Universitaires de France, 2013.

Simanca Pushaina, Estercilia, Por los valles de la arena dorada, Bogota, Ediciones Santillana, 2017.

Simanca Pushaina, Estercilia, « En las vísperas nacen las cerezas », Barranquilla, Lama

Producciones, 2019.

Simanca Pushaina, Estercilia, Entretien personnel, Riohacha, 29 juin 2017.

Viveiros De Castro, Eduardo, Metafísicas caníbales. Líneas de antropología postestructural, Madrid, Katz Editores, 2010.

\section{NOTES}

1. «Je t'offre alors La Guajira comme terre d'asile, dans la ranchería la plus abandonnée, où on boit de l'eau salée et où on mange de temps à autre [...] Pour que tu vives dans ta propre chair les promesses non tenues. Où peu nous importera ce que tu as fait ou ce que tu n'as pas fait puisque nous ne te le demanderons pas. [...] Dans cette ranchería ils ne pourront pas venir te chercher ni voudront le faire puisqu'ils savent que nous ne livrons pas ceux qui cherchent asile ici et puisqu'ils savent que cette soif de vie interminable, seuls peuvent la supporter les wayuu ». Je traduis cette citation et toutes celles des nouvelles de Simanca.

2. Avant les années 1970 , un certain nombre de transcriptions et de traductions des mythes avait eu lieu, notamment celles réalisées par l'intellectuel colombien Jorge Isaacs en 1884. Voir Guerra Curvelo Weildler, "El otro Isaacs", Revista entornos, 19 juin 2019. Disponible sur: http:// revistaentornos.com/el-otro-isaacs/. Consulté le 8 juin 2020.

3. Ceci est en lien avec la complexe histoire de mobilisations sociales des peuples indigènes en Colombie. Voir Laurent, Virginie, Comunidades indígenas, espacios políticos y movilización electoras en Colombia, 1990-1998. Motivaciones, campos de acción e impactos, Bogotá, IFEA, ICANH, 2005.

4. Les membres de la communauté étaient jusque-là désignés sous le nom générique de " guajiros".

5. Une ranchería est une habitation traditionnelle wayuu.

6. La littérature d'Estercilia Simanca est étudiée dans des travaux qui traitent de la littérature indigène en Colombie dans son ensemble, c'est le cas particulièrement des publications critiques de Miguel Rocha Vivas.

7. Pour un panorama général de la théorie décoloniale voir le numéro qui lui est consacré dans le Cahier des Amériques latines sous la coordination de Capucine Boidin et de Fátima Hurtado López. Boidin, Capucine et Hurtado López, Fatima, Cahier des Amériques Latines: Philosophie de la libération et tournant décolonial , 62, 2009.

8. Nous adoptons ici la définition que retiennent Gilles Deleuze et Félix Guattari de l'État (Deleuze G. et Guattari F., 1972), qu'ils conçoivent non seulement comme un appareil institutionnel mais aussi en termes de subjectivation politique. Le pouvoir de l'État se traduirait aussi dans les relations entre individus et groupes sociaux. Voir à ce propos, Sibertin Blanc, Guillaume, Politique et État chez Deleuze et Guattari. Essai sur le matérialisme historico-machinique, Paris, Presses Universitaires de France, 2013.

9. «Ce jour-là j'ai appris que mon oncle Tanko Pushaina s'appelait Tarzan, que Shankarit s'appelait Máximo, que Jutpunachón s'appelait Priscila, que Yaya s'appelait Clara, que Castorila 
s'appelait Cosita Rica, que Kawalashiyú s'appelait Marquesa, qu'Anawachón s'appelait John F. Kennedy, qu'Ashaneish s'appelait Cabeza [...] que le cousin Rafael Pushaina s'appelait Raspahierro, que mon cousin Matto s'appelait Bolsillo, et le temps d'un instant j'ai eu peur que ce soit également mon cas ».

10. Simanca Pushaina, Estercilia, « "Manifiesta no saber firmar" Nacido 31 de diciembre », Por los valles de arena dorada, Bogotá, Santillana, 2017, p. 26.

«[...] ils m'ont baptisée le 5 septembre 1970 et ma date de naissance 31 décembre 1965. Le document disait que je n'étais pas la fille de Korouna Pushaina ni de Colenshi Jusayú, mais de María Santa Pushaina avec Domingo Santo Jusayú et que je ne m'appelais pas Coleima Pushaina mais Faride Abuchaibe; que tous les enfants baptisés ce jour-là s'appelaient Faride et Eduardo Abuchaibe. Maintenant je comprends pourquoi tout le monde me surnomme la turque ».

11. Simanca Pushaina, Estercilia, «En las vísperas nacen las cerezas », Barranquilla, Lama Producciones, 2019, p. 9.

«À force d'accepter sans trop savoir ce qu'on nous demandait, nous avons commencé à célébrer de manière différente la fin de l'année, puisqu'un jour quelconque nous sommes tous "Nés un 31 décembre". L'État a oublié le nom des autres lunes et a voulu que comme célébration de fin d'année nous fêtions aussi notre anniversaire ».

12. Ce type de renversement correspond à ce que Miguel Rocha Vivas nomme "visiones de cabeza ", notion dont l'objectif est de comprendre les représentations que se font les communautés indigènes de la majorité nationale. D'après Rocha une "visión de cabeza » ne cherche pas à décrire une inversion dichotomique des relations de pouvoir politique où le dominé prendrait la place du dominant, il s'agit plutôt de nommer des renversements qui cherchent à construire d'autres perspectives interculturelles en révélant l'action des communautés à l'heure de penser la majorité nationale. Voir Rocha Vivas, 2016, op.cit., p. 24-27.

13. Simanca Pushaina, 2017, op.cit., p. 23.

"Cette fois-ci ils ont amené des papiers immenses qui avaient l'image de cet homme qui s'appelle "Candidat". Ils ont des noms étranges, ce ne serait pas bizarre que ce monsieur s'appelle ainsi. [...] Il y a une autre maison qui s'appelle Mairie et celui qui y habite s'appelle Maire, mais au départ il s'appelait comme l'autre... Candidat. Ne savent-ils pas qu'autant de noms peuvent entrainer de la confusion?».

14. "Cette transgression doit venir de quelqu'un de la communauté et non du fait que je sois allée à Madrid et que je sois revenue avec des idées libératrices pour ma communauté. Je suis transgressive puisque je suis wayuu, il est vrai que la femme wayuu doit avoir plus de libertés, mais la femme wayuu a déjà des libertés et elle les acquiert au fur et à mesure qu'elle acquiert une indépendance économique comme c'est le cas en Occident également. Lorsqu'en Europe on parle de mouvements féministes des années 80 , des années 70 , des années 60 , dans la communauté wayuu existaient déjà les femmes Julaima [...]».

15. Ibid., p. 90.

«Pendant un an Iiwa rêva de la jeune fille inconnue qui lui révélait avec ses mains et sans prononcer un seul mot, de plus en plus de secrets du tissage wayuu. [...] Lors du dernier rêve avec la jeune fille inconnue, puisqu'elle n'en a plus jamais eu, Iiwa se souvint de la légende de Waleker et découvrit que cette jeune fille était la même qui s'était transformée en araignée une fois découverte par son protecteur. Le chasseur qui la sauva en la trouvant seule et désemparée dans la brousse l'avait adoptée et amenée dans sa ranchería, et pour le remercier, quand personne ne la voyait, la jeune fille tirait des fils de sa bouche et réalisait des beaux tissages pour le chasseur. Une nuit se voyant découverte par lui, elle se transforma en araignée et fuit vers un arbre. Dès lors, elle fut transformée en Waleker, en araignée ».

16. Ibid., p. 42.

«Alors que j'attache un hamac dans la enramada pour que maman se repose, elle me dit qu'elle attendra le jour où son nombril tombera pour l'emporter et l'enterrer au pied du cerisier. Maman 
touche mon ventre et dit que je suis très fertile, non seulement pour mettre au monde ce bultito qui pleure mais aussi parce que les animaux qu'ils ont donnés pour moi ont accouché avant que l'année se soit écoulée, maman est uniquement venue pour recevoir ce qui lui est dû après mon accouchement $»$.

Une enramada est un lieu de réunion qui sert également pour la réalisation des rituels wayuu. 17. Les tuumas correspondent à la cornaline, il s'agit d'une pierre de protection pour les wayuu. 18. «La Julamia s'est rendu compte que l'immensité de sa fortune était aussi grande que le mot amour. Beaucoup d'hommes l'attendaient, ils lui rendaient visite désormais. [...] Elle maîtrisait son destin, elle chercherait son mari dans d'autres territoires, elle ferait une course de chevaux et ce serait elle qui fournirait la dot en échange du meilleur et du plus jeune des cavaliers ».

19. «Mon animal totémique n'a rien à voir avec le clan qui apparaît dans mon acte de naissance, [...] Mon totem concerne cet animal dur à chasser qui court avec son propre charme, il est souvent chassé et on dit que sa viande est délicieuse, je ne l'ai jamais goutée et je doute que je le fasse un jour, je suis comme mon grand-père qui n'a jamais mangé sa propre viande, le cochon ou le zaino, "Je ne peux pas me manger moi-même" il m'a dit. J'ai été blessée de nombreuses fois, mais j'ai su cicatriser, avec ma propre salive, les blessures de ce cerf que je suis... Oui, je suis Irama ». Je traduis.

20. Ibid, p. 97.

«Mon nom est Mireya. C'est ainsi que m'a nommée une marraine arabe que je n'ai plus jamais revue, mais tout le monde m'appelle Irama derrière mon dos, cela ne m'embête pas puisque je le suis. Parce que je ne suis pas passée par l'enfermement, mon esprit de petite fille est resté attaché à moi, je ne sais pas cuisiner et je ne vais pas apprendre, je n'aime plus avoir les cheveux longs et mes bijoux sont mieux dans la múcura qu'autour de mon cou, je ne marche pas derrière mes frères mais à leurs côtés, je ne sais pas garder silence, mais je sais garder les secrets, ceux-là m'accompagnent toujours ». Une múcura correspond à une pièce de poterie wayuu.

21. Entretien personnel avec Estercilia Simanca Pushaina, Riohacha, 29 juin 2017.

«[...] je suis une femme Irama, je ne suis pas passée par l'enfermement. Lorsqu'une femme wayuu ne passe pas par l'enfermement elle est comparée à un cerf, c'est-à-dire éternellement infantile et rebelle, une femme Irama, un peu folle ». Je traduis.

22. Voir, Simanca Pushaina, Estercilia « Daño emergente, lucro cesante », ibid, p. 65-67.

23. L'influence de l'univers afro-colombien est surtout visible, d'après l'auteure, dans le caractère oral de sa littérature.

24. Simanca Pushaina, 2019, op.cit., p. 7.

«Aujourd'hui comme jamais je me suis souvenue des paroles [de ma grand-mère]: "C'est beau quand le papier parle avec toi” (lire). Elle ne m'a pas parlé de la Mère Nature, elle m'a parlé de territoire, puisqu'où que j'aille je ne suis qu'une petite extension de mon cordon ombilical enterré au pied du cerisier dans la ranchería de mes grands-parents. Elle ne m'a pas appris à parler avec la lune ni avec le soleil, uniquement puisque je suis certaine que chaque pluie c'est mon anniversaire et chaque lune je paye mes impôts ». Je traduis.

25. Pour plus de détails voir, Simanca Pushaina, Estercilia «Pulowi de Üchimüin » in Duchesne Winter Juan, Hermosos invisibles que nos protegen, Pittsburgh, Instituto Internacional de Literatura Iberoamericana, 2015, p. 319-321.

26. «À quel moment les blancs lui donnent l'étiquette de contrebande ? Ce que nous faisons est simplement un échange et c'est un dialogue de l'Occident avec le peuple wayuu, et le peuple wayuu prend d'Occident ce qui lui sert, ce qui lui convient ».

27. Entretien personnel avec Estercilia Simanca Pushaina, Riohacha, 29 juin 2017.

« [être latino-américain c'est] être plus universel mais en langue espagnole, amener la réalité du peuple auquel tu appartiens à tous les hispanophones, et que quelqu'un du Guatemala te dise, "ça arrive aussi dans ma communauté" et celui qui te dit cela ne doit pas être nécessairement indigène ". 


\section{RÉSUMÉS}

Cet article se propose de penser le caractère négociateur de la poétique d'Estercilia Simanca Pushaina afin de démontrer que sa littérature, en écriture alphabétique conventionnelle et en espagnol, traduit en profondeur les épistémologies - fondées sur la tradition orale - de la communauté indigène wayuu, installée dans la péninsule de La Guajira, entre la Colombie et le Venezuela. Cela revient à penser la littérature de Simanca à la lumière de l'histoire, en particulier du phénomène de la contrebande économique qui traverse la communauté indigène wayuu depuis le $\mathrm{XVII}^{\mathrm{e}}$ siècle. Récupérer l'historicité de la littérature de Simanca signifie mettre au centre la capacité d'action politique de l'auteure et de la communauté wayuu. En effet, Simanca rend compte d'une communauté multiple qui ne peut pas être rattachée à une identité culturelle fixe : chez les wayuu la réinvention culturelle constitue la clé qui permet d'assurer leur autonomie politique. La littérature de Simanca témoigne de ces stratégies de réinvention culturelle et de négociation politique : elle invite au dialogue avec la société colombienne majoritaire tout en mettant en avant la profondeur de la conception wayuu de la temporalité et les relations politicosociales qui en découlent.

This article seeks to explore the negotiation character of Estercilia Simanca Pushaina's poetics with the purpose of demonstrating that her literature, in conventional alphabetic writing and in Spanish, translates in depth the epistemologies - based on the oral tradition - of the indigenous wayuu community, installed in the peninsula of La Guajira, between Colombia and Venezuela. This means studying Simanca's literature in the light of history, concerning, in particular the phenomenon of economic smuggling that characterizes the wayuu indigenous community since the $17^{\text {th }}$ century. Recovering the historicity of Simanca's literature means putting the author's and the wayuu community's capacity of political agency at the center of the discussion. In her literature, Simanca gives an account of a multiple community that cannot be reduced to a fixed cultural identity: in the wayuu community, cultural reinvention is the key to ensuring its political autonomy. Simanca's poetics testify to these strategies of cultural reinvention and political negotiation by opening a dialogue with the Colombian majoritarian society and highlighting the profound conception of temporality in the wayuu community and the sociopolitical relations that emerge from there.

Este artículo busca pensar el carácter de negociación de la poética de Estercilia Simanca Pushaina con el propósito de demostrar que su literatura, en escritura alfabética convencional y en español, traduce en su profundidad las epistemologías - fundadas en la tradición oral - de la comunidad indígena wayuu, instalada en la península de La Guajira, entre Colombia y Venezuela. Esto significa pensar la literatura de Simanca a la luz de la historia, en particular del fenómeno de contrabando económico que atraviesa la comunidad indígena wayuu desde el siglo xVII. Recuperar la historicidad de la literatura de Simanca significa poner en el centro de la discusión la capacidad de acción política de la autora y de la comunidad wayuu. En efecto, Simanca da cuenta de una comunidad múltiple que no puede ser reducida a una identidad cultural fija: en la comunidad wayuu la reinvención cultural constituye la clave que permite asegurar su autonomía política. La literatura de Simanca da testimonio de estas estrategias de reinvención cultural y negociación política, invita al diálogo con la sociedad colombiana mayoritaria poniendo de realce la concepción profunda de la temporalidad en la comunidad wayuu y las relaciones sociopolíticas que de ahí emergen. 


\section{INDEX}

Mots-clés : Communauté wayuu, littérature alphabétique contemporaine, réinvention culturelle, émancipation politique

Keywords : Wayuu community, contemporary alphabetic literature, cultural reinvention, political emancipation

Palabras claves : Comunidad wayuu, literatura alfabética contemporánea, reinvención cultural, emancipación política

\section{AUTEUR}

\section{LAURA LEMA SILVA}

Laura Lema Silva est doctorante en études latino-américaines à l'Université Lumière Lyon 2, laboratoire LCE (Lettres et Civilisations Étrangères EA 1853). Ses recherches explorent l'apport des structures de connaissance wayuu au concept d'émancipation politique à travers la littérature alphabétique conventionnelle de la communauté publiée à partir des années 1970. Auteure du chapitre : «José Carlos Mariátegui's Artistic Criticism for a Broader Approach to Aesthetics Emancipatory Potential », in Peter Baker, Irina Feldman, Mike Geddes, Felipe Lagos y Roberto Pareja, Latin American Marxisms in context. Past and Present, Cambridge Scholars Publishg, 2019. Adresse mail : lauralema.s@gmail.com 